\title{
Social selection of game organizers promotes cooperation in spatial public goods games
}

Yongkui Liu

Xiaojie Chen (chenx@iiasa.ac.at)

Lin Zhang

Fei Tao

Long Wang

\section{Approved by}

Ulf Dieckmann

Director, Evolution and Ecology Program

June 2015 


\title{
Social selection of game organizers promotes cooperation in spa- tial public goods games
}

\author{
Yongkui Liu ${ }^{1,2}$, Xiaojie $\mathrm{Chen}^{3}$, Lin Zhang ${ }^{1}$, Fei TaO ${ }^{1}$ and Long Wang ${ }^{4}$ \\ 1 School of Automation Science and Electrical Engineering, Beihang University - Beijing 100191, China \\ 2 School of Electronic and Control Engineering, Chang'an University - Xi'an 710064, China \\ 3 Evolution and Ecology Program, International Institute for Applied Systems Analysis (IIASA) - Schlossplatz 1, 2361 \\ Laxenburg, Austria \\ 4 Center for Systems and Control, State Key Laboratory for Turbulence and Complex Systems, College of Engineering, \\ Peking University - Beijing 100871, China
}

PACS $02.50 . \mathrm{Le}$ - Decision theory and game theory
PACS $87.23 . \mathrm{Ge}$ - Dynamics of social systems
PACS $89.75 . \mathrm{Fb}$ - Structures and organization in complex systems

\begin{abstract}
Inspired by the fact that opportunities in reality are heterogeneous for individuals due to social selection, we propose an evolutionary public goods game model considering the social selection of game organizers occurring on a square lattice. We introduce a simple rule that, depending on the value of a single parameter $\mu$, influences the selection of players that are considered as potential game organizers. For positive $\mu$ players with a high payoff will be considered more likely. Setting $\mu$ equal to zero returns the random selection of game organizers. We find that increasing the probability of selecting the wealthier individuals as game organizers can effectively promote cooperation. We show that the promotion of cooperation attributes to the dominance of the clusters of cooperative organizers in the population by investigating the evolution of spatial patterns.
\end{abstract}

Introduction. - Cooperation within groups of selfish individuals is ubiquitous in human and animal societies. Evolutionary game theory provides a powerful framework to explain the emergence of cooperation among selfish individuals [1-3]. The evolutionary public goods game (PGG) in particular has attracted considerable attention in previous studies [4-6]. In the public goods game, players decide simultaneously whether to contribute to a common pool or not. The total contribution is multiplied by a synergy factor which is larger than one. Then the resulting public goods are equally shared among all the participating players, no matter whether they contribute. It is clear that it is best to defect (not contribute), yet the collective interests are maximized when all individuals cooperate, which results in the "tragedy of the commons" [7].

Since the pioneering work of Nowak and May [8], spatial games have received much attention [9]. In the framework of spatial games, ingredients such as the social diversity $[10,11]$, population density [12], variable multiplication factor [13], optional participation [14,15], threshold $[16,17]$, risk $[4,18]$ and continuous strategy [19-21] have been considered. In addition, much efforts have been directed toward the exploration of the effects of personal features on cooperation, such as punishment [22-28], reward and reputation [29-32], to name but a few. Other ingredients, such as noise [33] have also been considered (see Ref. [34] for a very recent review on the public goods game).

Quite remarkably, in the early investigations, it has been discovered that heterogeneity can greatly elevate the survivability of cooperators in the PGG, no matter the heterogeneity is associated with group size [10] or with other factors, e.g., preferential selection [11]. On the other hand, in most of previous studies, it is conventionally assumed that the chance for organizing the game is equal, which actually overlooks the role of social selection since in the real world such chance is often closely related with one's wealth (or influence). Different individuals may have different amounts of wealth and influences, the chance may therefore be different. For example, individuals are more willing to participate in a social activity held by a wealthy or an influential individual since the participation may be 
more profitable, which reflects the role of social selection. Inspired by this idea, we consider a simple addition to the public goods game that allows only a fraction of players to organize the game, i.e., introducing the propensity of designating the most successful individuals as the game organizers. Is this beneficial for the evolution of cooperation? The answer is not straightforward and it would be significant and also interesting to answer this question.

Comparing with previous works the propensity of designating the most successful individuals as game organizers is the most significant difference. Our aim is to study how the social selection of game organizers affects the evolution of cooperation in the spatial PGG. By means of systematic computer simulations we demonstrate that assigning preferentially only a fraction of individuals the chance for organizing the game can remarkably promote cooperation in the spatial PGG.

Model. - We consider a spatial public goods game among a population of individuals distributed over a square lattice of size $L \times L$ with periodic boundary conditions. Each individual occupies one site of the lattice and no empty site exists. The evolution of the game is iterated in accordance with the simulation procedure comprising the following elementary steps. First, every individual plays the games within its von Neumann neighbourhood. Next, all individuals are allowed to modify their strategies simultaneously by imitating a randomly chosen neighbour's strategy.

Before proceeding to the interaction stage, we first select the game organizers. That is, in each time step, a fraction $f(0<f \leq 1.0)$ of individuals are selected as the potential game organizers. For the sake of improving efficiency, we adopt the following sequential selection algorithm. That is, we select the organizers in sequence until the number of game organizers reaches $f \times L \times L$. After each time of selection, the selected individual is excluded from the population. The probability for individual $i$ to be selected as an organizer is

$$
\Pi_{i}=\frac{\exp \left(\mu P_{i}\right)}{\sum_{z} \exp \left(\mu P_{z}\right)}
$$

where $P_{i}$ is the overall payoff of individual $i$ obtained in the previous round of the game, and the sum runs over all individuals at the moment when the selection is performed. Initially, all individuals' payoffs are set to zero. The selection parameter $\mu$ is introduced to govern the weight of the payoff in the organizer selection process. Here we assume $\mu \geq 0$ considering that social selection favors wealthy individuals. Evidently, for $\mu=0$ the organizers are chosen uniformly at random among the entire population. For $\mu>0$, however, a preference toward the individuals who have a higher payoff is introduced. Note that $f=1.0$ recovers the conventional model [10].

After selecting the game organizers, each individual plays the PGG within its von Neumann neighborhood. That is, if an individuals is an organizer, it can organize a PGG involving it itself and its four direct neighbors. Moreover, it can also participate in the PGGs organized by the direct neighbors. If there are no individuals having the chance to organize a game within its neighborhood, then nothing happens. In the PGG interactions, each individual decides whether to contribute to a common pool. Cooperators contribute a fixed amount of investment 1.0, while defectors contribute nothing. The total contribution is multiplied by a synergy factor $r>1$ and then is shared equally among all players, no matter whether they contribute or not. Thus, the payoff of player $i$ per PGG is $p_{i}=r \frac{n_{i}}{N}-1\left(p_{i}=r \frac{n_{i}}{N}\right)$ if it is a cooperator (defector), where $r$ is the synergy factor of the game, $n_{i}$ denotes the number of cooperators in group $i$, and $N=5$ represents the size of group $i$, i.e., the number of participants in the group. The overall payoff of each player is accumulated from all PGG interactions, that is $P_{i}=\sum_{z} p_{z}$, where the sum runs all groups of PGG interactions of $i$.

After playing the games, each individual is allowed to update strategy by learning a randomly chosen neighbor. The probability for individual $i$ to imitate a randomly chosen neighbor $j$ is

$$
W_{j \rightarrow i}=\frac{1}{1+\exp \left[\left(P_{i}-P_{j}\right) / \kappa\right]}
$$

where $P_{i}$ and $P_{j}$ denote the overall payoffs of $i$ and $j$, respectively, and $\kappa$ introduces some noise to allow for irrational choices, which is simply set to 0.1 for all simulations.

The evolution of the system starts from the initial setup with $50 \%$ cooperators and $50 \%$ defectors who are homogeneously dispersed over the square lattice. The key quantity for characterizing the system is the density of cooperators $\rho_{C}$ among the population, which is the fraction of cooperators in the entire population. We implement the model with synchronous strategy update. Results of computer simulations presented below are obtained for a population comprising $50 \times 50$ individuals. The density of cooperators $\rho_{C}$ is obtained by averaging over 100 realizations and for each realization, we average 10000 generations after a transient time of 1000000 generations where the dynamical equilibrium has been reached. It is worth pointing out that for $\mu \geq 1$, the density of cooperators in a single realization of evolution is either 0 or 1 . Thus, $\rho_{C}$ there is actually the probability for the system to reach full $C$ state instead of the actual cooperation level, which is obtained by calculating the runs which lead to full cooperation among the total runs.

Results. - We start by presenting the stationary density of cooperators in dependence on the synergy factor $r$, as shown in fig. 1. It can be observed that cooperation is overall remarkably enhanced for $f<1.0$ as compared with that for $f=1.0$. The promotion of cooperation is more pronounced for smaller $f$ and with increasing $f$, the results increasingly move toward that for $f=1.0$. $\mu$ can also have an effect on the emergence of cooperation. When $f$ is small, small values of $\mu$ may result in a lower cooperation 


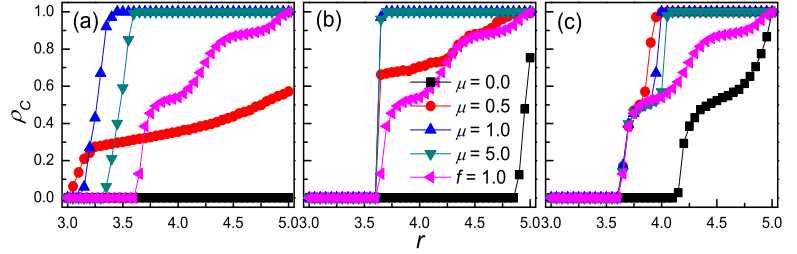

Fig. 1: Density of cooperators $\rho_{C}$ in dependence on $r$ for different values of $\mu$ at different values of $f$ : (a) $f=0.2$, (b) $f=0.5$, (c) $f=0.8$. For comparison, the result for $f=1.0$ where all individuals have the chance to hold the game is presented.

level [see panel (a)], whereas as $f$ increases, small values of $\mu$ become increasingly more beneficial to cooperation [see panel (c)]. Moreover, cooperation for smaller values of $\mu$ arises at smaller values of $r$. In particular, for $\mu=0.0$, cooperation can emerge only when $f$ is sufficiently large. These results suggest that assigning individuals heterogeneous chance to hold the public goods game can effectively promote the evolution of cooperation. In what follows, we will systematically investigate the roles of $f$ and $\mu$ in the evolution of cooperation, respectively.

Figure 2 shows the density of cooperation as a function of $f$. It can be observed that for intermediate values of $\mu$, cooperators can be optimally favored at an intermediate value of $f$. For sufficiently high values of $\mu$, full cooperation can be achieved even at very small values of $f$ (e.g., $f=0.02)$ and $\rho_{C}$ declines with increasing $f$. While for $\mu=0.0$, cooperators can survive only when both $f$ and $r$ are very high. It is worth noting that cooperation for larger values of $\mu$ ascends and descends at smaller values of $f$ in comparison with that for small values of $\mu$. From fig. 3 , one can find that preferential selection of game organizers $(\mu>0.0)$ is the key for the promotion of cooperation.

In fig. 3, we show the density of cooperators as a function of $\mu$. When $r$ is small, small values of $f$ are more favorable to cooperation, and there exists an intermediate value of $\mu$ best favoring the evolution of cooperation. As $r$ increases, the phenomenon of optimal cooperation occurring at intermediate values of $\mu$ happens for larger values of $f$, and cooperation for smaller values of $f$ tends to increases monotonically. Furthermore, with increasing $r$, cooperation for larger values of $f$ can be higher than that for smaller values of $f$. Interestingly and surprisingly, a local minimum of $\rho_{C}$ occurs when $r$ is large (which will be explained later).

Thus far, we have demonstrated the effects of the parameters $f$ and $\mu$, respectively. The underlying mechanism, however, is unclear. To this end, we investigate the time evolution of spatial patterns focusing on the effects of $f$ and $\mu$, as shown in figs. 4 and 5 . Let us first look at fig. 4 which concentrates on the effect of $f$. Initially, the four types of players randomly distribute over the square lattice [see panels (a), (f), (k)]. However, due to the different values of $f$, the evolutionary trends are quite different. For small values of $f$, the cooperative organizers have the

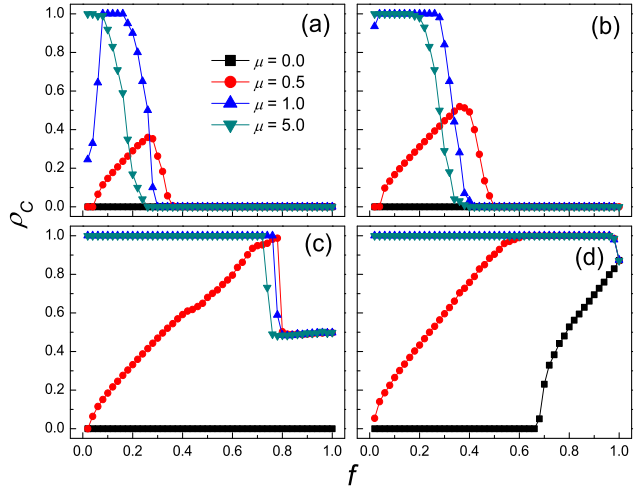

Fig. 2: Density of cooperators $\rho_{C}$ in dependence on the fraction of organizers $f$ for four different values of $\mu$ at four different values of $r$ : (a) $r=3.4$, (b) $r=3.6$, (c) $r=3.8$, and (d) $r=4.5$.

chance to unite each other and lead the system to full cooperation. Eventually, a single cluster comprising all cooperative organizers is established. While for large values of $f$, the cooperative organizers are usually besieged by the defecting organizers and have no chance to expand their territories. Eventually, cooperators disappear. It is worth noting that the established cluster of cooperative organizers [see figs. 4(e) and (j)] is dynamically drifting over the square lattice instead of being stationary due to the stochasticity in the game organizer selection process.

One can find that the core mechanism for the promotion of cooperation has two key ingredients: the formation of cooperative organizer clusters and the random drift at the interface separating the cooperative and defecting non-organizers. The formation of the cooperative organizer cluster is attributed to the current organizer selection method which favors individuals with higher payoffs. Cooperators can collect higher payoffs by forming clusters, which makes them to be selected as game organizers with a high probability. The promotion of cooperation can be attributed to the leadership of the clusters of cooperative organizers. The random drift is supported by the current Fermi strategy updating rule for which the probability for the cooperative and defecting non-organizers (whose payoffs are both zero) to learn each other is 0.5. Both factors guarantee the success of cooperators.

Besides the evolutionary outcome, the time scale for the evolution is also noteworthy. One can find that with increasing $f$, the evolutionary time scales for different values of $f$ are distinct. Specifically, as $f$ increases, the time for the system to reach the stationary state reduces. For example, for $f=0.02,0.2$ and 0.8 , the time steps for the system to reach the stationary state are approximately 160000, 1300 and 120, respectively. The above phenomenon can be easily understood. When $f$ is small, the size of the established cooperative organizer cluster is small, thus the surface of the contact between the cooperative and defecting non-organizers is small, which makes 


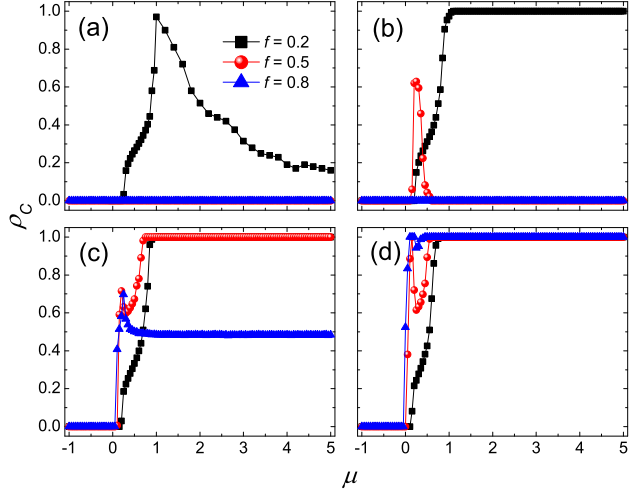

Fig. 3: Density of cooperators $\rho_{C}$ in dependence on the selection parameter $\mu$ for different values of $f$ at four different values of $r$ : (a) $r=3.4$, (b) $r=3.6$, (c) $r=3.8$, and (d) $r=4.5$.

the transient process become longer.

In what follows, we return to the effect of $f$ on cooperation. We must understand that what determines the final evolutionary outcome is the competition between cooperative and defecting organizers. Varying $f$ leads to the change in the density of organizers and thus changes the comparative advantages between cooperative and defecting organizers. The rationale is as follows. When $f$ is large (consider the extreme case of $f=1.0$ ), most individuals among the population are game organizers. The case, to a large extent, returns to the traditional case where cooperation is comparatively unfavorable since at the boundary defectors usually have advantages over cooperators in collecting payoffs [37]. When $f$ is very small, the game organizers usually isolatedly dispersed on the square lattice which is favorable for a cooperative organizer to expand its territory since the menace coming from the adjacent defecting organizers can be avoided. On the other hand, the small number of cooperative organizers in turn increases the probability for them to be eliminated. Another key problem is that even the small cooperative organizer cluster can be formed, the time for the system to reach full cooperation may be very long. While for intermediate values of $f$, the cooperative organizers have a high survival probability, and at the same time, due to the cooperative and defecting organizers have comparatively low probabilities to encounter each other, the direct menace coming from the latter is small. All these lead to the flourish of cooperation in the population.

In fig. 5 we focus on the role of $\mu$ in the evolution of cooperation. According to our game organizer selection rule, the stochasticity in the game organizer selection decreases with increasing $\mu$, that is, with increasing $\mu$, individuals with higher payoffs are more likely to be selected as game organizers. According to the above rationale, when $\mu$ is small, the game organizers are almost selected randomly. This cannot guarantee that the selected individuals have high payoffs. Therefore, the selected cooperative orga-

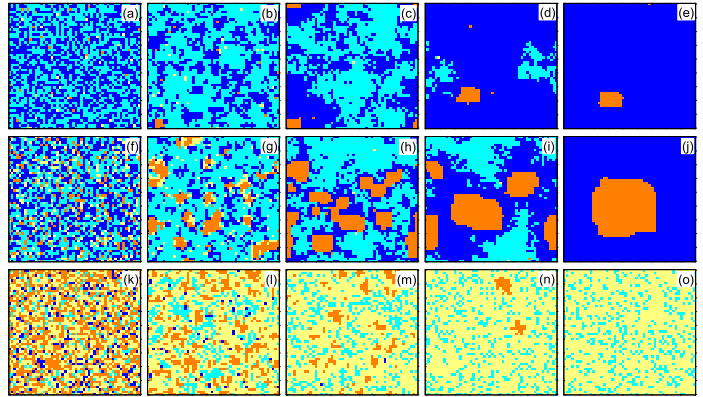

Fig. 4: Time evolution of spatial patterns of cooperative organizers (orange), cooperative non-organizers (blue), defecting organizers (light yellow) and defecting non-organizers (cyan) for $f=0.02\left[(\mathrm{a}) t=0, \rho_{C}=0.5\right.$, (b) $t=10, \rho_{C}=0.3546$, (c) $t=1000, \rho_{C}=0.4736$, (d) $t=156000, \rho_{C}=0.9224$, (e) $\left.t=160000, \rho_{C}=1.0\right], f=0.2\left[(\mathrm{f}) t=0, \rho_{C}=0.5\right.$, (g) $t=5$, $\rho_{C}=0.3344$, (h) $t=30, \rho_{C}=0.5024$, (i) $t=200, \rho_{C}=0.7036$, (j) $\left.t=1300, \rho_{C}=1.0\right], f=0.8\left[(\mathrm{k}) t=0, \rho_{C}=0.5\right.$, (l) $t=2$, $\rho_{C}=0.2212,(\mathrm{~m}) t=4, \rho_{C}=0.082,(\mathrm{n}) t=20, \rho_{C}=0.0184$, (n) $\left.t=120, \rho_{C}=0.0\right]$. Other parameters are $r=3.6, \mu=1.0$.

nizers cannot shoulder the role of leaders and lead to the spreading of cooperation. For high values of $\mu$, individuals with high payoffs are almost selected with certainty. Since cooperators in the clusters of cooperative organizers and the boundary defecting organizers (who collect payoffs by exploiting cooperators) usually have high payoffs, they are more likely to be selected, as shown in fig. $5(\mathrm{j})$. Since the cooperative organizers are completely surrounded by the defecting ones, and the selected defecting organizers have highest payoffs, the cooperative organizers are gradually eliminated. While for intermediate values of $\mu$, one the one hand, the selection is not random, and on the other hand, the selected defecting organizers do not necessarily have the highest payoffs (as can be seen from fig. 5(f) where a number of isolated defecting organizers are selected). As another effect resulting from the stochasticity, the defecting organizers attached to the cooperative organizers are not always selected. This makes there exist gaps surrounding the cooperative organizer clusters. All these ingredients lead to the promotion of cooperation.

In our model, $f$ denotes the density of organizers in the population, and it thus determines the chance for cooperative and defecting organizers to meet each other. While $\mu$ determines the weight of payoffs in the selection of organizers. One can find from figs. $5(\mathrm{j})$ and $(\mathrm{k})$ that increasing $\mu$ makes the defecting organizers who are adjacent to the cooperative organizers be selected with a high probability, which is unfavorable to cooperation. Therefore, for high values of $\mu$, in order for the promotion of cooperation, the chance for the cooperative and defecting organizers to encounter each other should be small, which corresponds to small $f$. This is why the region of $f$ where cooperation is promoted for increasing $\mu$ moves toward left.

It remains to explain the local minimum of $\rho_{C}$ shown in figs. 3(c) and (d). We take the case of $r=4.5, f=0.5$ 


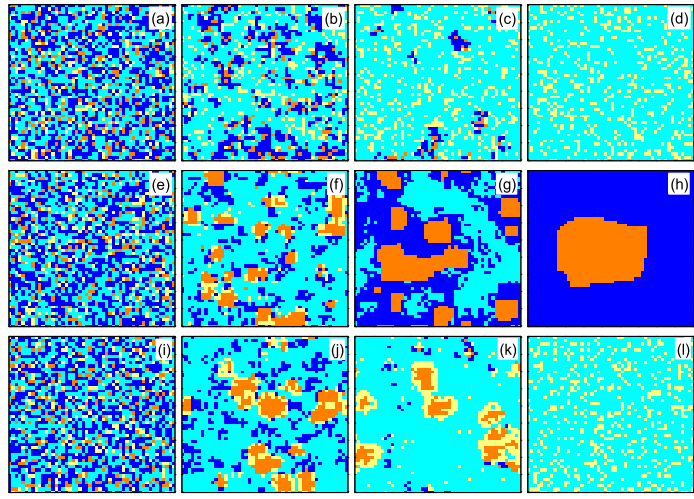

Fig. 5: Time evolution of spatial patterns of cooperative organizers (orange), cooperative non-organizers (blue), defecting organizers (light yellow) and defecting non-organizers (cyan) for $\mu=0.0\left[\right.$ (a) $t=0, \rho_{C}=0.5$, (b) $t=4, \rho_{C}=0.286$, (c) $t=20, \rho_{C}=0.054$, (d) $\left.t=50, \rho_{C}=0.0\right], \mu=1.2[(\mathrm{e}) t=0$, $\rho_{C}=0.5$, (f) $t=10, \rho_{C}=0.3052,(\mathrm{~g}) t=60, \rho_{C}=0.6592,(\mathrm{~h})$ $\left.t=1000, \rho_{C}=1.0\right], \mu=5.0\left[(\mathrm{i}) t=0, \rho_{C}=0.5,(\mathrm{j}) t=10\right.$, $\rho_{C}=0.296$, (k) $t=38, \rho_{C}=0.0896$, (l) $\left.t=100, \rho_{C}=0.0\right]$ [see fig. 3(a)]. Other parameters are $r=3.4, f=0.2$.

for example. It can be observed from fig. 3(d) that the density of cooperators for $f=0.5$ first increases to 1.0 at $\mu=0.15$ and then drops to 0.62 at $\mu=0.25$. After that, the density of cooperators reverts to 1.0 again quickly. Similar phenomena can also be observed in fig. 3(c). Note also that such phenomenon can only be observed for large values of $r$. Moreover, the phenomenon occurs at small values of $\mu$. Here we also resort to the spatial patterns to explore the underlying reason for the phenomenon, as shown in fig. 6. One can find that different values of $\mu$ lead to distinct stationary spatial patterns. Since the selection of game organizers depends both on $\mu$ and individual payoff $P$ (which in turn depends on the value of $r)$, investigation of the distribution of $\exp (\mu P)$ may be helpful for our analysis. The results are shown in fig. 7 . One can find that since $r=4.5$ is large (which results in high payoffs of individuals), small variations of $\mu$ can lead to great changes of $\exp (\mu P)$. For example, although $\mu=0.25$ has small change with respect to $\mu=0.15$, the change of $\exp (\mu P)$ is large. Consequently, the effect of random selection for $\mu=0.15$ is strong while for $\mu=0.25$, the randomness of selection of game organizers is weak. The spatial patterns are closely correlated with the extent of the stochasticity. For $\mu=0.15$, due the stochasticity, the cooperators in cooperator clusters are not necessarily selected while the cooperative non-organizers in the last time step may be selected, which results in the pattern shown in fig. 6(a). While for $\mu=0.25$, individuals with higher payoffs are more likely to be selected such that the cooperative organizers can form a large cluster with the cooperative non-organizers lying at the interface separating the cooperators and defectors. Since there exist defecting organizers in the defector areas, the boundary cooperative non-organizers are not capable to invade the

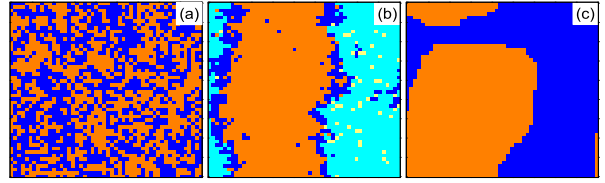

Fig. 6: Stationary spatial patterns for $r=4.5, f=0.5$, and (a) $\mu=0.15$, (b) $\mu=0.25$, and (c) $\mu=1.0$, respectively.

defectors, which establishes an equilibrium between the cooperators and defectors. While for $\mu=1.0$, the randomness of the organizer selection is very weak. At this moment, we should emphasize the large value of $r$, which gives cooperators more chance to survive. In combination with large values of $r$ which favors cooperators, the cooperative organizers can wipe out the defecting ones and eventually dominate the population. It must be aware that the large values of $r$ play a crucial role. Large values of $r$ make individuals have high payoffs such that even small change in $\mu$ (i.e., from 0.15 to 0.25 ) leads to large change of $\exp (\mu P)$. This is why the local minimum occurs at small values of $\mu$. One the other hand, large values of $r$ favor cooperators. If $r$ is small, the cooperative organizers may be eliminated by the neighboring defecting ones.

Discussions and conclusion. - The promotion of cooperation in the present work can be attributed to the heterogeneity in organizing the pubic goods games resulting from the social selection. The difference in individuals' organizing chance leads to the heterogeneity in the number of PGGs in which individuals participate. The present mechanism responsible for the promotion of cooperation is similar to that reported in Ref. [10] where the authors investigated the public goods game taking place on the scale-free networks. In Ref. [10], individuals also participate in different number of PGGs due to individuals have different number of neighbors.

It is also worth noting that in Ref. [12], the authors studied how the population density affects the evolution of cooperation in the spatial public goods game occurring on a square lattice. The population density there corresponds to the fraction of game organizers here and they have similar effects on the evolution of cooperation although the mechanisms facilitating cooperation are different.

Note also that here the payoff in the last time step is used for determining the organizing chance. In the real society, however, one's wealth can be better characterized by its accumulative payoff $[35,36]$. We have checked that when organizers are selected according to the accumulative payoff, cooperation can be better favored. Due to the intrinsic mechanism for the promotion of cooperation, the population size and the strategy updating rule can also have effects on the evolution of cooperation. Regarding the population size, we found that smaller population size can better promote cooperation for small values of $\mu$ (e.g., $\mu<1.0)$. For large values of $\mu$, the difference of the density of cooperators for different population sizes is small. 


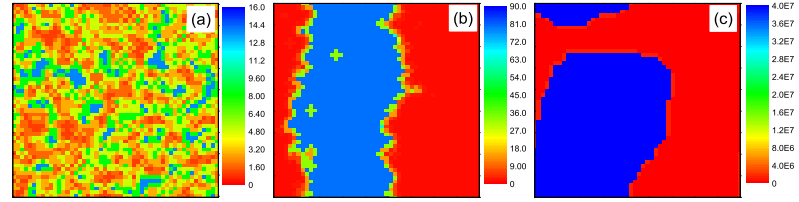

Fig. 7: Stationary distribution of $\exp (\mu P)$ on the square lattice for $r=4.5, f=0.5$, and (a) $\mu=0.15$, (b) $\mu=0.25$, and (c) $\mu=1.0$, respectively.

Concerning the strategy updating rule, we also apply the "proportional imitation" rule and the "choosing the best" rule. It is found that these two rules, cooperation can be promoted mainly for small values of $r$. An important difference of these two rule from the currently employed Fermi rule is that the latter supports the imitation between individuals with the same payoff while the former do not, which may be responsible for the inhibition of cooperation for the "proportional imitation" rule and the "choosing the best" rule at large values of $r$.

In summary, we have studied the evolution of cooperation in the spatial public goods game with the social selection of organizing chance which results from the payoffbased preferential selection. We have shown that cooperation can be significantly promoted when only a fraction of preferentially selected individuals are assigned the chance of organizing public goods games. Our results elucidate the effect of social selection of organizing chance on the evolution of cooperation in the spatial public goods games, which appears to enable the tragedy of the commons to be avoided.

\section{$* * *$}

Helpful discussions with X.-S Zhang are gratefully acknowledged. This work was supported by National Natural Science Foundation of China (NSFC) under grant No. 61203374, 61074144, 51005012, 61103096, 10972002, and 11161011.

\section{REFERENCES}

[1] Axelrod R., The Evolution of Cooperation (Basic Books, New York) 1984

[2] Sмiтh J. M., Evolution and the Theory of Games (Cambridge University Press, Cambridge, UK) 1982.

[3] NowA M. A., Evolutionary Dynamics: Exploring the Equations of Life (Harvard University Press, Cambridge, England) 2006.

[4] Santos F. C. and Pacheco J. M., Proc. Natl. Acad. Sci. USA, 108 (2011) 10421.

[5] Greenwood G., EPL, 95 (2011) 40006.

[6] Heitzig J., Lessman K. and Zou Y., Proc. Natl. Acad. Sci. USA, 108 (2011) 15737.

[7] Hardin G., Science, 162 (1968) 1243.

[8] NowaK M. A. and MaY R. M., Nature (London), 359 (1992) 826.

[9] Szabó G. and Fáth G., Phys. Rep., 446 (2007) 97.
[10] Santos F. C., Santos M. D. and Pacheco J. M., Nature (London), 454 (2008) 213.

[11] Yang H.-X., Wang W.-X., Wu Z.-X., LAI Y.-C. and Wang B.-H., Phys. Rev. E, 79 (2009) 056107.

[12] Wang Z., Szolnoki A. and Perc M., Phys. Rev. E, 85 (2012) 037101.

[13] Chen X., Liu Y., Zhou Y., Wang L. and Perc M., Phys. Rev. E, 7 (2012) e36895.

[14] Szabó G. and Hauert C., Phys. Rev. Lett., 89 (2002) 118101.

[15] Hauert C., De Monte S., Hofbauer J. and Sigmund K., Science, 296 (2002) 1129.

[16] Szolnoki A. and Perc M., Phys. Rev. E, 81 (2010) 057101.

[17] Boza G. and SzÁmadó S., BMC Evol. Biol., 10 (2010) 336.

[18] Chen X., Szolnoki A. and Perc M., EPL, 99 (2012) 68003.

[19] Kamimura Y., Aвe J. and Ito H., J. Theor. Biol., 252 (2008) 277.

[20] Cressman R., Song J. W., Zhang B. Y. and Tao Y., J. Theor. Biol., 299 (2012) 144.

[21] Chen X., Szolnoki A., Perc M. and Wang L., Phys. Rev. E, 85 (2012) 066133.

[22] Fowler J. H., Proc. Natl. Acad. Sci. USA, 102 (2005) 7047.

[23] Brandt H., Hauert C. and Sigmund K., Proc. Natl. Acad. Sci. USA, 103 (2006) 495.

[24] Traulsen A., Hauert C., Silva H. D., Nowak M. A. and Sigmund K., Proc. Natl. Acad. Sci. USA, 106 (2009) 709.

[25] Helbing D., Szolnoki A., Perc M. and Szabó G., New J. Phys., 12 (2010) 083005.

[26] Sigmund K., Silva H. D., Traulsen A. and Hauert C., Nature (London), 466 (2010) 861.

[27] Szolnoki A., Szabó G. and Czakó L., Phys. Rev. E, 84 (2011) 046106.

[28] Rand D. G. and NowaK M. A., Nature Communications, 2 (2011) 434.

[29] Rand D. G., Dreber A., Ellingsen T., Fudenberg D. and NowaK M. A., Science, 325 (2009) 1272.

[30] Szolnoki A. and Perc M., EPL, 92 (2010) 38003.

[31] Hauert C., J. Theor. Biol., 267 (2010) 22.

[32] Sasaki T. and Unemi T., J. Theor. Biol., 287 (2011) 109.

[33] Szolnoki A., Perc M. and Szabó G., Phys. Rev. E, 80 (2009) 056109.

[34] Perc M., Gómez-Gardeñes J., Szolnoki A., Floría L. M. and Moreno Y., J. R. Soc. Interface, 10 (2013) 20120997.

[35] Liu Y.-K., Li Z., Chen X.-J. and Wang L., Chin. Phys. $B, 19$ (2010) 090203.

[36] Chadefaux T. and Helbing D., PLoS ONE, 5 (2010) e13471.

[37] Chen X., Fu F. and Wang L., Phys. Rev. E, 78 (2008) 051120 